\title{
THE
}

6-2013

\section{Antipsychotic Prescribing Pathways, Polypharmacy, and Clozapine Use in Treatment of Schizophrenia}

Jessica L. Gören

University of Rhode Island, jgoren@challiance.org

Meterko

Sandra Williams

Gary J. Young

Errol Baker

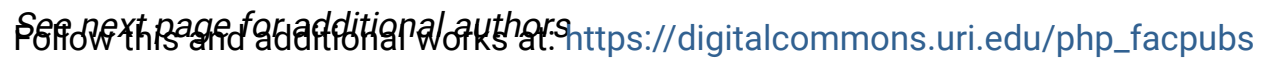

The University of Rhode Island Faculty have made this article openly available. Please let us know how Open Access to this research benefits you.

This is a pre-publication author manuscript of the final, published article.

Terms of Use

This article is made available under the terms and conditions applicable towards Open Access

Policy Articles, as set forth in our Terms of Use.

\section{Citation/Publisher Attribution}

Gören, J. L., Meterko, M., Williams, S., Young, G. J., Baker, E., Chou, C.H., Kilbourne, A. M., \& Bauer, M. S. (2013). Antipsychotic Prescribing Pathways, Polypharmacy, and Clozapine Use in Treatment of Schizophrenia. Psychiatric Services, 64(6), 527-533. doi: 10.1176/appi.ps.002022012

Available at: http://dx.doi.org/10.1176/appi.ps.002022012 
Authors

Jessica L. Gören, Meterko, Sandra Williams, Gary J. Young, Errol Baker, Chia-Hung Chou, Amy M. Kilbourne, and Mark S. Bauer

This article is available at DigitalCommons@URI: https://digitalcommons.uri.edu/php_facpubs/66 


\section{Antipsychotic Prescribing Pathways, Polypharmacy, and Clozapine Use in Treatment of Schizophrenia}

Jessica L. Gören, PharmD

Mark Meterko, PhD

Sandra Williams, MS

Gary J. Young, JD, PhD

Errol Baker, PhD

Chia-Hung Chou, PhD

Amy M. Kilbourne, PhD, MPH

Mark S. Bauer, MD

Dr. Gören is affiliated with the Department of Pharmacy Practice, University of Rhode Island, Kingston (e-mail: jgoren@challiance.org address 1035 Cambridge St, Suite 23, Cambridge, MA 02141).

Dr. Gören, Dr. Meterko, Ms. Williams, Dr. Young, Dr. Baker, and Dr. Bauer are with the Veterans Affairs (VA) Center for Organization, Leadership and Management Research, VA Boston Healthcare System, Boston.

Dr. Young is with the Center for Health Policy and Healthcare Research and Dr. Chou is with the School of Pharmacy, both at Northeastern University, Boston.

Dr. Kilbourne is with the Health Services Research and Development Service, Center for Clinical Management Research, VA Ann Arbor, Ann Arbor, Michigan. 
Objective: To ensure optimal care for patients with schizophrenia, antipsychotic medications must be appropriately prescribed and used. Therefore, the primary objectives of this study were to identify and describe pathways for antipsychotic prescribing, assess the consistency of observed pathways with treatment guidelines, and describe variability across facilities.

Methods: Data from Veterans Affairs administrative data sets from fiscal year (FY) 2003 to FY 2007 were gathered for analysis in this retrospective cohort study of antipsychotic prescribing pathways among 13 facilities across two regional networks. Patients with a new episode of care for schizophrenia or schizoaffective disorder in FY 2005 were identified, and antipsychotic prescribing history was obtained for two years before and after the index diagnosis.

Demographic characteristics and distribution of comorbidities were assessed. Median medical center rates of polypharmacy were calculated and compared with Fisher's exact test.

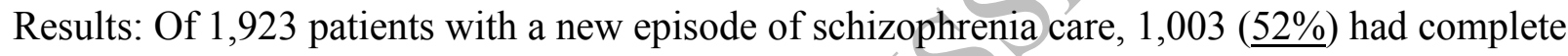
data on prescribing pathways. A majority (74\%) of patients were prescribed antipsychotic monotherapy, and 19\% received antipsychotic polypharmacy. Of patients receiving antipsychotic polypharmacy, 65\% began polypharmacy within 90 days of starting any antipsychotic treatment. There was a fourfold difference in polypharmacy across facilities. Antipsychotic polypharmacy was not associated with geographic location or medical center patient volume. Clozapine utilization was low $(0 \%-2 \%)$.

Conclusions: Retrospective examination of longitudinal prescribing patterns identified multiple antipsychotic prescribing pathways. Although most patients received guideline-concordant care, antipsychotic polypharmacy was commonly used as initial treatment, and there was substantial variability among facilities. Study findings suggest the utility of secondary data to assess treatment adaptation or switching for practical clinical trials.

Although antipsychotics are effective for the treatment of schizophrenia, many patients do not respond to the first antipsychotic prescribed (1-3). Patients without a response on a second antipsychotic trial are typically considered treatment resistant (4-10). Because clozapine is the only antipsychotic proven effective for treatment-resistant schizophrenia, it is widely recognized as the preferred treatment option for patients who have not responded to two antipsychotic 
monotherapy trials (4-10). This approach is reflected in evidence-based treatment guidelines, which recommend that such patients be offered clozapine (6-10).

With these evidence-based treatment guidelines, one can conceptualize guideline-concordant treatment as an antipsychotic (AP) prescribing pathway, whereby $\mathrm{AP} 1 \rightarrow \mathrm{AP} 2 \rightarrow$ clozapine. However, studies indicate that adherence to this guideline is low (11-25). Increasingly, longterm use of two or more regularly scheduled antipsychotics (polypharmacy) is prescribed, often before clozapine $(11,15,17-19,23-25)$. This practice is inconsistent with evidence-based guidelines, which do not endorse long-term antipsychotic polypharmacy and recommend it only as a last-line treatment when clozapine has failed (6-10).

Rates of clozapine and antipsychotic polypharmacy use vary by treatment setting (11-25). However, most studies to date on antipsychotic prescribing have been cross-sectional even though longitudinal assessment is required to characterize appropriate use of polypharmacy. Cross-sectional studies cannot make the important distinction between antipsychotic polypharmacy use for treatment-resistant individuals (such as after a failed clozapine trial) and for other, less discriminating use. Also, findings from cross-sectional studies do not provide necessary information to assess which patients may be eligible for clozapine $(11,16,20)$. This is of particular importance because the longitudinal studies that have been conducted indicate that clozapine is started after the failure of an average of six antipsychotic trials, including a period of antipsychotic polypharmacy, suggesting that the most effective treatment for treatment-resistant patients is delayed or not used $(17-19,24,25)$.

Longitudinal studies of large administrative data sets are becoming increasingly important. The development of more cost-efficient adaptive and practical clinical trials could be informed by data from existing sources. Also, studies examining the impact of policy or implementation strategies on prescribing pathways can be done in a naturalistic setting, guiding development of interventions that may be successful in real-world applications. Ultimately these studies may improve use of guideline-based treatment and outcomes in mental health.

Therefore the goals of this descriptive retrospective cohort study were to use administrative data to develop a systematic method for describing longitudinal antipsychotic prescribing pathways and to describe the observed sequence of antipsychotic trials as related to evidencebased guideline recommendations. 


\section{Methods}

\section{Setting and data sources}

The Veterans Health Administration (VHA) is a national health care system treating $>87,000$ veterans with schizophrenia or schizoaffective disorder annually. Because the VHA has used electronic records for clinical care for many years, readily available administrative data sets can be used to identify and describe longitudinal antipsychotic prescribing pathways. Administrative data have been used extensively in other antipsychotic studies $(11,20,26-28)$.

We focused on 13 medical centers across two Veterans Affairs (VA) Integrated Service Networks (VISNs). We chose two VISNs in different regions of the United States that serve a large number of veterans with schizophrenia or schizoaffective disorder. Data to identify our target sample and characterize individuals' medication history were obtained from the VA National Patient Care Database (NPCD), which contains information on inpatient and outpatient visits, ICD-9-CM diagnoses and procedure (CPT-4) codes, dispensed medication, and patient demographic characteristics. Data on average patient volume were derived from the fiscal year (FY) 2004 VA National Psychosis Registry (NPR) report, which includes data from multiple sources for all veterans treated for psychoses within the VHA.

\section{Study sample}

We identified all patients from two VISNs who received a prescription for an antipsychotic and were actively engaged in ongoing treatment, with a new episode of care for spectrum disorders in FY 2005. We focused on new episodes of care because treatment recommendations begin with the first treatment chosen for an episode of illness.

A new episode of care was defined as an ICD-9-CM schizophrenia-related diagnosis (codes 205.0-295.9) in at least one inpatient or two outpatient encounters in FY 2005, no schizophreniarelated diagnosis in the previous two years, initiation of an index antipsychotic prescription, and no antipsychotic prescription for daily use $>90$ days in the year before the diagnosis. Patients with multiple psychiatric disorders coded on the diagnosis date were included if the number of schizophrenia-related diagnoses exceeded or equaled the number of other psychiatric disorders (20). Patients had to be actively engaged in VHA treatment, as evidenced by at least one VHA 
outpatient visit for any medical or psychiatric disorder in both FY 2006 and FY 2007. The Boston VHA institutional review board approved this study.

\section{Antipsychotic use}

Antipsychotic use was defined as any dose of an antipsychotic (American Hospital Formulary Service class 28:16.08) for daily use dispensed from an outpatient VHA pharmacy for $\geq 90$ days in FY 2005. The 90-day criterion was chosen on the basis of previous studies in which discontinuation of treatment due to adverse events versus inefficacy occurred earlier in treatment (25).

The index antipsychotic prescription was defined as the first antipsychotic prescribed for daily use in FY 2005. To minimize the likelihood of falsely categorizing as polypharmacy transitions from one antipsychotic to another, we defined polypharmacy as concomitant prescription of two antipsychotics for daily use for $>90$ days (11). Intermittent exposures of $\leq 90$ days were considered noninformative.

\section{Informative sample}

Each patient's prescribing pathway was characterized according to the antipsychotic trials received during the two years after the index antipsychotic trial. As summarized in Figure 1, receipt of a single antipsychotic other than clozapine for daily use (pathway 1) or two sequential prescriptions for regularly scheduled nonclozapine antipsychotics (pathway 2) with no gaps $>90$ days for the duration of the study was categorized as monotherapy. Receipt of two prescriptions concomitantly for $>90$ days wăs categorized as polypharmacy. Antipsychotic polypharmacy could occur at various points in the treatment sequence (pathways 3-6). Patients who received clozapine for daily use as the second or third monotherapy trial were categorized as receiving early clozapine (pathway 8) or clozapine (pathway 7), respectively. Patients who received three or more sequential nonclozapine antipsychotics were categorized as delayed use of clozapine (pathway 9). Because prescription history data were available to definitively characterize pathways $1-9$, we refer to them collectively as informative cases.

\section{Noninformative cases}

Some observed pathways were noninformative because incomplete information made a categorization ambiguous or uncertain. For example, some cases received a single trial (pathway 
11) or sequential trials of antipsychotics (pathway 10) but no further antipsychotic prescriptions during the observation period. Thus it was not possible to characterize these pathways.

\section{Characterization of antipsychotic pathways}

Pathways 1, 2, and 7 represent guideline-endorsed antipsychotic prescribing pathways and thus were characterized as guideline concordant. In cases of extreme suicidality or aggression, guidelines endorse using clozapine as the second antipsychotic, so pathway 8 was categorized as guideline concordant (6). Pathways 3-6 all involved polypharmacy before clozapine was prescribed, and these pathways were categorized as guideline nonconcordant. Antipsychotic polypharmacy including clozapine was considered guideline nonconcordant because clozapine monotherapy was not used before polypharmacy was initiated. Pathway 9 involved use of a nonclozapine antipsychotic after two failed antipsychotic trials and was categorized as nonconcordant with guidelines. Pathways with clozapine as the first antipsychotic were excluded from the study because these likely reflect treatment-resistant patients for whom standard guideline-concordant care may not be appropriate.

\section{Analytic sample}

Previous studies have demonstrated that antipsychotic monotherapy and polypharmacy are the most common forms of antipsychotic treatment (12-14). These antipsychotic prescribing pathways also have the clearest relationship to guideline recommendations. Hence these groups formed the analytic sample and were used to address the second major aim of the study, comparison of prescribing practices with treatment guidelines. Because patients requiring clozapine treatment represent either more severely ill or treatment-resistant populations, they were excluded from the analytic sample.

\section{Measures and analyses}

Our measures and analyses proceeded at both the patient and facility center levels. At the patient level, our primary dependent variable was pathway assignment. Using the $I C D-9-C M$ diagnosis codes associated with each medical encounter during the study, we created presence and absence variables for the antipsychotic-related comorbidities diabetes and hyperlipidemia. From the NPCD we obtained five demographic characteristics: age at time of the index visit, marital status 
(four categories), gender, self-reported race (U.S. Census categories), and self-reported ethnicity (whether, yes-no, patients considered themselves Hispanic, Latino, or Spanish).

We used basic frequencies, central tendency, and variance statistics to describe the number of cases in each of the antipsychotic pathway groups as well as the demographic and comorbidity profiles of all informative cases and the analytic sample. Within the analytic sample, we compared the demographic characteristics and distribution of comorbidities between monotherapy and polypharmacy groups by computing chi square statistics on the crosstabulation of antipsychotic pathway (monotherapy and polypharmacy) by those categorical variables. For age, we computed independent-groups $t$ tests between monotherapy and polypharmacy.

At the medical center level, we computed a polypharmacy rate as the percentage of cases in the analytic sample at that medical center that fell into the monotherapy and polypharmacy pathways. The denominator for this rate was the total number of cases in our analytic sample: the combined monotherapy and polypharmacy groups. As a measure of the total volume of patients with severe mental illness (psychotic and bipolar disorders), we obtained counts of such cases for each medical center from the NPR. Finally, because facilities were located in two geographically diverse VISNs, a dichotomous categorical location code was created to represent region (designated A or B). For analyses at the medical center level, we used basic descriptive statistics to describe the distribution of polypharmacy rates.

To examine the association between polypharmacy rate and geographic region, we computed the median polypharmacy rate at the medical center level, classified facilities as above or below that rate, and computed the Fisher exact probability test on the cross-tabulation of polypharmacy rate (below or above median) by region. Finally, to examine the association between polypharmacy rate and volume of patients with severe mental illness, we computed the facility median for yolume of these patients, classified facilities as being above or below that volume, and then computed the Fisher exact probability test on the cross-tabulation of polypharmacy rate (below or above median) and volume of patients with severe mental illness (below or above median).

\section{Results}


We identified 1,923 new treatment episodes of schizophrenia and schizoaffective disorders in FY 2005 and their distribution across nonredundant pathway groups (Table 1). Of the total, 310 (16\%) veterans did not utilize VHA outpatient services in FY 2006 and FY 2007. Another 338 (18\%) utilized VHA services but did not receive antipsychotic medications from an outpatient VHA pharmacy in FY 2005, and 272 (14\%) did not receive a sufficient number of antipsychotic prescriptions to allow reasonable judgment about appropriate pathway assignment. These 920 noninformative cases (48\%) were not included in further analyses. There was no difference in median age between the informative and noninformative groups.

The remaining 1,003 informative cases involved patients who entered a new treatment episode for schizophrenia or schizoaffective disorder, were treated with an antipsychotic in FY 2005-FY 2007 and had one or more outpatient clinic visits in FY 2006 and FY 2007. Within the informative sample, 763 patients $(76 \%)$ received care consistent with treatment guidelines (Table 1). The most common guideline-concordant prescribing pathway was monotherapy $(\mathrm{N}=745$, $74 \%)$, followed by early use of clozapine $(\mathrm{N}=17,2 \%)$. One patient received clozapine after two antipsychotic trials. The remaining $24 \%(\mathrm{~N}=240)$ of informative cases followed prescribing pathways that were nonconcordant with guidelines, with nonclozapine polypharmacy being the most common path $(\mathrm{N}=188,19 \%)$. Notably, of patients receiving antipsychotic polypharmacy, $65 \%(\mathrm{~N}=127)$ received polypharmacy as their first antipsychotic treatment and $4 \%(\mathrm{~N}=45)$ received polypharmacy in place of clozapine.

A total of 933 informative cases (93\%) qualified for the analytic sample, which for reasons discussed above consisted of the (nonclozapine) antipsychotic monotherapy $(\mathrm{N}=745)$ and (nonclozapine) polypharmacy $(\mathrm{N}=188)$ pathway groups. Self-reported race and ethnicity were missing in $49 \%$ and $51 \%$ of cases, respectively. The descriptive statistics for these variables and the chi square analyses were based, respectively, on persons whose race $(\mathrm{N}=474)$ or ethnicity $(\mathrm{N}=454)$ was known. The analytic sample was predominantly single (45\%), Caucasian (54\%), and male (93\%) (Table 2), with a mean \pm SD age of $50.7 \pm 12.2$. The incidence of diabetes and hyperlipidemia was $16 \%$ and $17 \%$, respectively.

There were no statistically significant associations between pathway group and demographic characteristics or comorbidities, with three exceptions. Six percent of the nonclozapine monotherapy sample was female compared with $13 \%$ of the nonclozapine_polypharmacy group 
$\left(\chi^{2}=9.39, \mathrm{df}=1, \mathrm{p}<.01\right)$, and $52 \%$ of the monotherapy group was Caucasian compared with $65 \%$

of the polypharmacy group $\left(\chi^{2}=8.54, \mathrm{df}=3, \mathrm{p}<.05\right)$. A significant association was observed with a higher proportion of hyperlipidemia in the polypharmacy group (22\%) compared with the monotherapy group $\left(15 \% ; \chi^{2}=4.38, \mathrm{df}=1, \mathrm{p}<.05\right)$.

We observed a more than fourfold difference in antipsychotic polypharmacy rates between the medical centers (Figure 2). The median medical center polypharmacy rate was $21 \%$, and the likelihood of being above that rate was no greater in region A compared with region $\mathrm{B}(\mathrm{p}=.59$, by Fisher exact test). The median annual medical center volume of cases of severe mental illness was 1,616 , and the likelihood of being above or below the median polypharmacy rate for a facility was no greater at lower-volume facilities compared with higher-volume facilities $(p=.10$, by Fisher exact test).

\section{Discussion}

Using retrospective data of longitudinal antipsychotic prescribing, we classified 1,003 of 1,923 patients $(52 \%)$ with new treatment episodes of schizophrenia into one of 11 antipsychotic prescribing pathways. Of these informative cases, $74 \%$ were treated along antipsychotic monotherapy pathways, consistent with treatment guidelines. This is consistent with estimates that $70 \%-80 \%$ of patients with schizophrenia are responsive to treatment $(1,2)$.

We found that $24 \%$ of patients received care nonconcordant with guidelines, primarily involving antipsychotic polypharmacy. A total of $13 \%$ of informative cases received polypharmacy before receiving a monotherapy antipsychotic trial. This rate is 3.5 times higher than previously reported in the California Medicaid population (29). This discrepancy is most likely related to methodological differences. Whereas the Medicaid study reported antipsychotic polypharmacy as the initial treatment for patients who received prescriptions for two antipsychotics at the first fill, our study defined polypharmacy as initial treatment when patients did not receive an adequate monotherapy trial of antipsychotic before receiving antipsychotic polypharmacy. Thus, for our population, initial treatment with antipsychotic polypharmacy included patients who received polypharmacy within the first 90 days of treatment. The fact that $65 \%$ of patients treated with polypharmacy started antipsychotic polypharmacy within the first 90 days of treatment indicates that a majority of patients on polypharmacy were not treated with a single evidence-based treatment. In summary, both studies suggest that antipsychotic 
polypharmacy is often being used as the initial treatment, contravening all evidence-based guidelines.

Similar to other studies, our study showed a significant difference among medical centers in utilization of antipsychotic polypharmacy, and this variability was not associated with geographic region or volume of patients with severe mental illness. Previous studies indicated that clinicians reserve antipsychotic polypharmacy for patients with more severe illness. However, it seems unlikely there would be a fourfold difference in the number of severely ill patients between facilities and that $65 \%$ of patients receiving polypharmacy were so sick that they required antipsychotic polypharmacy as first-line treatment (30). Thus future research should investigate whether organizational characteristics account for some variability in antipsychotic prescribing. For example, differences in availability of resources, staffing, quality improvement capability, program implementation, care coordination, and communication may affect provision of care (26,31-33). The organization and provision of care have been shown to vary among VHA mental health programs, which may contribute to the observed variation $(31,33)$.

Medical centers with lower volume of patients with severe mental illness trended toward higher rates of polypharmacy. Whereas five of six low-volume medical centers had higher rates of polypharmacy, five of seven high-volume medical centers had lower rates of polypharmacy, suggesting that more experience with severe mental illness may be related to increased adherence with treatment guidelines.

Clozapine underutilization is an issue in the treatment of schizophrenia and schizoaffective disorders, possibly because of increased prescribing burden, including prescriber and patient registration requirements; significant side-effect burden; and frequent laboratory testing to monitor for side effects. In this study, only about $2 \%(\mathrm{~N}=18)$ of patients in the informative sample received clozapine consistent with evidence based guidelines, whereas 45 patients who fared poorly on two antipsychotic trials did not receive clozapine. Some of these cases may reflect antipsychotic intolerance rather than clozapine nonresponse. However, given that up to $20 \%$ of patients with schizophrenia are treatment resistant whereas under $2 \%$ of patients received clozapine and almost 19\% received antipsychotic polypharmacy, it seems unlikely that use of 
clozapine is optimized. It is concerning that patients may be receiving polypharmacy in place of clozapine or that initiation of clozapine is delayed.

Consistent with other studies, receipt of antipsychotic polypharmacy was more common with women and Caucasian patients $(29,34)$. Our finding that patients receiving antipsychotic polypharmacy were more likely to have hyperlipidemia is contrary to at least one previous study (35). However, antipsychotic polypharmacy may be a marker for factors such as body mass index and concomitant medications that were not controlled for in this study.

It is interesting to note that 338 patients diagnosed as having schizophrenia or schizoaffective disorder received care within the VHA but did not receive antipsychotics. Some of these patients may fill their prescriptions outside the VHA. It is also possible that these patients had less severe illness or chose not to fill antipsychotic prescriptions and therefore represent a population in need of outreach.

\section{Conclusions}

To our knowledge, this study is unique in its description and categorization of antipsychotic prescribing pathways. Our method allowed for identification of justified deviations from treatment guidelines and of patients possibly eligible for a clozapine trial. Nonetheless, there are some limitations that should be noted. Data derived from prescription files do not account for nonadherent patients, and the filling of a prescription does not necessarily mean that the medications were taken how and when they should have been. Given that this study did not focus on treatment outcomes, however, this limitation is not a critical concern. Also, such data cannot account for patient and clinician preferences. Medication changes may have been secondary to drug intolerance rather than inefficacy, and thus all 45 patients with two unsuccessful sequential trials of monotherapy may not have been appropriate for a clozapine trial. However, the use of the 90-day minimum to identify an antipsychotic trial in this study makes this somewhat less likely.

Consistent with prior cross-sectional analyses, we observed that $19 \%$ of patients with schizophrenia treated within the VHA received polypharmacy, and the utilization of clozapine was several-fold lower. A large portion of patients started with polypharmacy as the initial antipsychotic trial. In addition, there was unexplained variability between medical centers in use of antipsychotic polypharmacy. Future research should consider the causes of variability and its 
impact on patient outcomes. Pathways analysis is a useful way to use secondary data to inform practical clinical trials and effectiveness of prescribing policies.

\section{Acknowledgments and disclosures}

This study was funded in part by a Mental Health Quality Enhancement Research Initiative Locally Initiated Project Fund 68-001 from the VA Health Services Research and Development Service and by IIR 10-314.

The authors report no competing interests. 


\section{References}

1. Dencker SJ: The need for long-term neuroleptic treatment in schizophrenia. Acta Psychiatrica Scandinavica Supplementum 291:29-43, 1981

2. Jäger M, Riedel M, Obermeier M, et al: Time course of antipsychotic treatment response in schizophrenia: results from a naturalistic study in 280 patients. Schizophrenia Research 118:183188,2010

3. Lieberman JA, Stroup TS, McEvoy JP, et al: Effectiveness of antipsychotic drugs in patients with chronic schizophrenia. New England Journal of Medicine 353:1209-1223, 2005

4. McIlwain ME, Harrison J, Wheeler AJ, et al: Pharmacotherapy for treatment-resistant schizophrenia. Journal of Neuropsychiatric Disease and Treatment 7:135 -149, 2011

5. Conley RR, Kelly DL: Management of treatment resistance in schizophrenia. Biological Psychiatry 50:898-911, 2001

6. American Psychiatric Association: Practice guideline for the treatment of patients with schizophrenia, 2nd ed. American Journal of Psychiatry 161(Feb suppl):1-56, 2004

7. National Institute of Health and Clinical Excellence: Core interventions in the treatment and management of schizophrenia in primary and secondary care (CG82). March 2009. Available at guidance.nice.org.uk/CG82. Accessed Dec 3, 2011

8. Kreyenbuhl J, Buchanan RW, Dickerson FB, et al: The Schizophrenia Patient Outcomes Research Team (PORT): updated treatment recommendations 2009. Schizophrenia Bulletin 36:94-103, 2010

9. Behavioral Healthcare Performance Measurement System Measures. Alexandria, Va, National Association of State Mental Health Program Directors Research Institute, March 2007. Available at www.nri-inc.org. Accessed Dec 3, 2011

10. Moore TA, Buchanan RW, Buckley PF, et al: The Texas Medication Algorithm Project antipsychotic algorithm for schizophrenia: 2006 update. Journal of Clinical Psychiatry 68:983990,2007

11. Kreyenbuhl J, Valenstein M, McCarthy JF, et al: Long-term combination antipsychotic treatment in VA patients with schizophrenia. Schizophrenia Research 84:90-99, 2006

12. Sernyak MJ, Rosenheck RA: Antipsychotic use in the treatment of outpatients with schizophrenia in the VA from fiscal years 1999 to 2006. Psychiatric Services 59:567-569, 2008

13. Grohmann R, Engel RR, Geissler KH, et al: Psychotropic drug use in psychiatric inpatients: recent trends and changes over time-data from the AMSP study. Pharmacopsychiatry 37(suppl 1):S27-S38, 2004 
14. Centorrino F, Goren JL, Hennen J, et al: Multiple versus single antipsychotic agents for hospitalized psychiatric patients: case-control study of risks versus benefits. American Journal of Psychiatry 161:700-706, 2004

15. Conley RR, Kelly DL, Lambert TJ, et al: Comparison of clozapine use in Maryland and in Victoria, Australia. Psychiatric Services 56:320-323, 2005

16. Fayek M, Flowers C, Signorelli D, et al: Psychopharmacology: underuse of evidence-based treatments in psychiatry. Psychiatric Services 54:1453-1454, 1456, 2003

17. Taylor DM, Young C, Paton C: Prior antipsychotic prescribing in patients currently receiving clozapine: a case note review. Journal of Clinical Psychiatry 64:30-34, 2003

18. Wheeler AJ: Treatment pathway and patterns of clozapine prescribing for schizophrenia in New Zealand. Annals of Pharmacotherapy 42:852-860, 2008

19. Schumacher JE, Makela EH, Griffin HR: Multiple antipsychotic medication prescribing patterns. Annals of Pharmacotherapy 37:951-955, 2003

20. VA National Psychosis Registry. Ann Arbor, Michigan US Department of Veterans Affairs, 2009

21. Bronskill SE, Gill SS, Paterson JM, et al: Exploring variation in rates of polypharmacy across long-term care homes. Journal of the American Medical Directors Association (Epub ahead of print, Aug 12, 2011)

22. Weissman EM: Antipsychotic prescribing practices in the Veterans Healthcare Administration-New York metropolitan region. Schizophrenia Bulletin 28:31-42, 2002

23. Harrison J, Janlöv M, Wheeler AJ: Patterns of clozapine prescribing in a mental health service in New Zealand. Pharmacy World and Science 32:503-511, 2010

24. Lee B, Walker V: Polypharmacy as the initial second-generation antipsychotic treatment. Psychiatric Services 59:717, 2008

25. Tsutsumi C, Uchida H, Suzuki T, et al: The evolution of antipsychotic switch and polypharmacy in natural practice — a longitudinal perspective. Schizophrenia Research 130:4046, 2011

26. McCarthy JF, Blow FC, Valenstein M, et al: Veterans Affairs Health System and mental health treatment retention among patients with serious mental illness: evaluating accessibility and availability barriers. Health Services Research 42:1042-1060, 2007 
27. Kreyenbuhl JA, Valenstein M, McCarthy JF, et al: Long-term antipsychotic polypharmacy in the VA health system: patient characteristics and treatment patterns. Psychiatric Services 58:489-495, 2007

28. Ren XS, Kazis LE, Lee AF, et al: Patient characteristics and prescription patterns of atypical antipsychotics among patients with schizophrenia. Journal of Clinical Pharmacy and Therapeutics 27:441-451, 2002

29. Gilmer TP, Dolder CR, Folsom DP, et al: Antipsychotic polypharmacy trends among Medicaid beneficiaries with schizophrenia in San Diego County, 1999-2004. Psychiatric Services 59:1007-1010, 2007

30. Sernyak MJ, Rosenheck R: Clinicians' reasons for antipsychotic coprescribing. Journal of Clinical Psychiatry 65:1597-1600, 2004

31. Kilbourne AM, Greenwald DE, Hermann RC, et al: Financial incentives and accountability for integrated medical care in Department of Veterans Affairs mental health programs. Psychiatric Services 61:38-44, 2010

32. Santone G, Bellantuono C, Rucci P, et al: Patient characteristics and process factors associated with antipsychotic polypharmacy in a nationwide sample of psychiatric inpatients in Italy. Pharmacoepidemiology and Drug Safety 20:441-449, 2011

33. Kilbourne AM, Pincus HA, Schutte K, et al: Management of mental disorders in VA primary care practices. Administration and Policy in Mental Health 33:208-214, 2006

34. Daumit GL, Crum RM, Guallar E, et al: Outpatient prescriptions for atypical antipsychotics for African Americans, Hispanics, and whites in the United States. Archives of General Psychiatry 60:121-128, 2003

35. Correll CU, Frederickson AM, Kane JM, et al: Does antipsychotic polypharmacy increase the risk for metabolic syndrome? Schizophrenia Research 89:91-100, 2007 
Figure 1 Antipsychotic (AP) prescribing pathways among veterans with schizophrenia or schizoaffective disorder ${ }^{\mathrm{a}}$

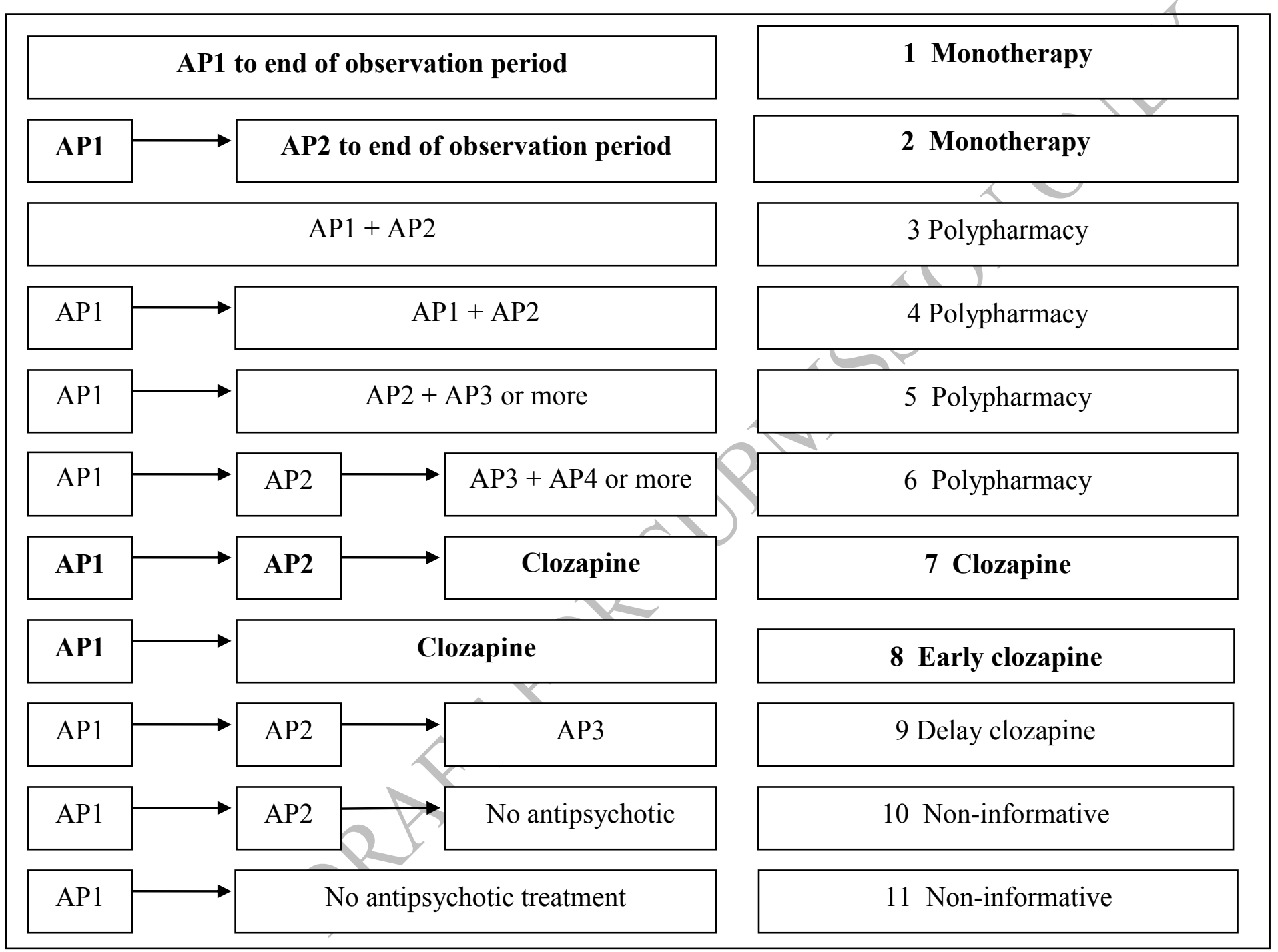

${ }^{\mathrm{a}}$ Guideline-concordant pathways are in boldface. 
Figure 2 Rates of polypharmacy for treatment of schizophrenia or schizoaffective disorder, by VHA facility ${ }^{\mathrm{a}}$

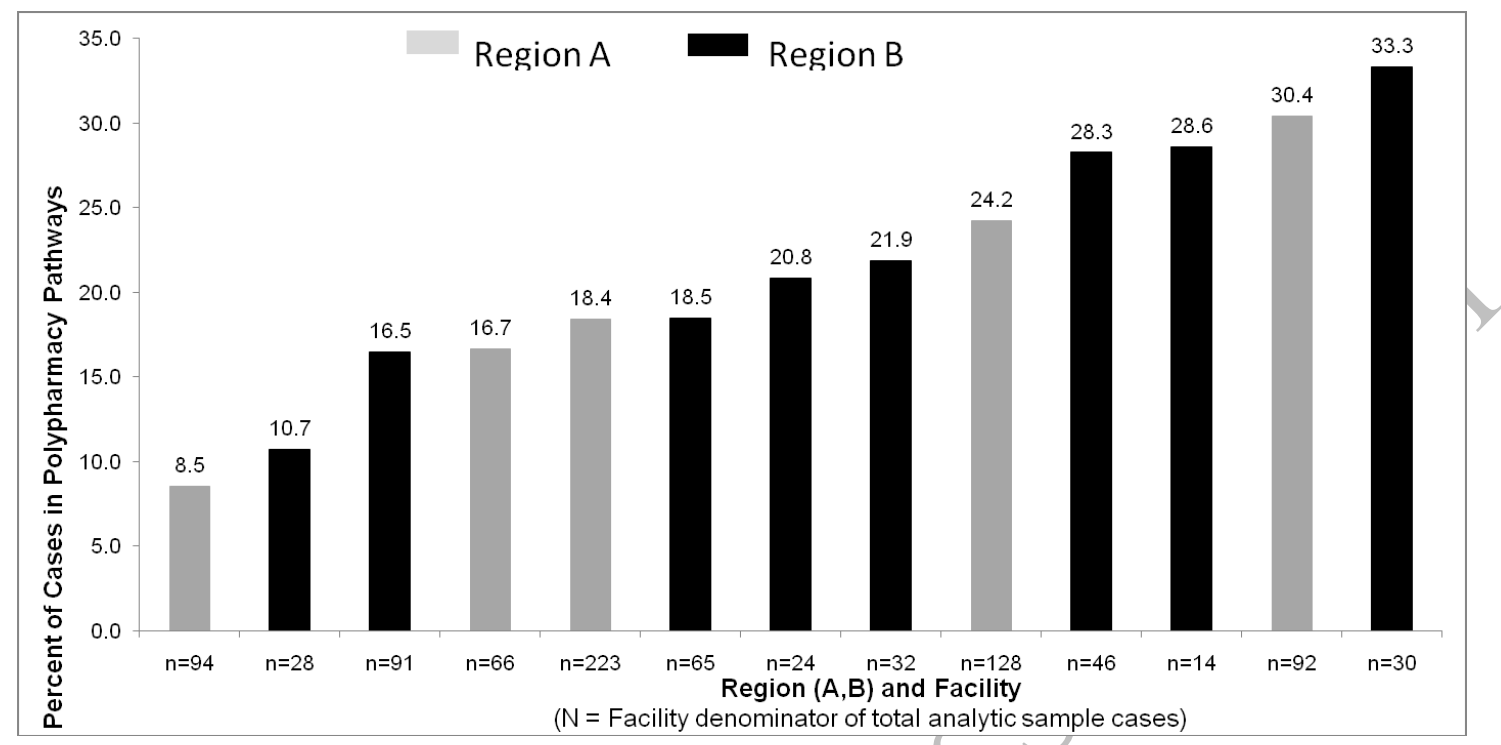

${ }^{a} \mathrm{~N}$ indicates the facility denominator of total analytic sample cases. VHA, Veterans Health Administration 
Table 1 Observed distribution of antipsychotic prescribing pathways for VHA patients with schizophrenia or schizoaffective disorder ${ }^{\mathrm{a}}$

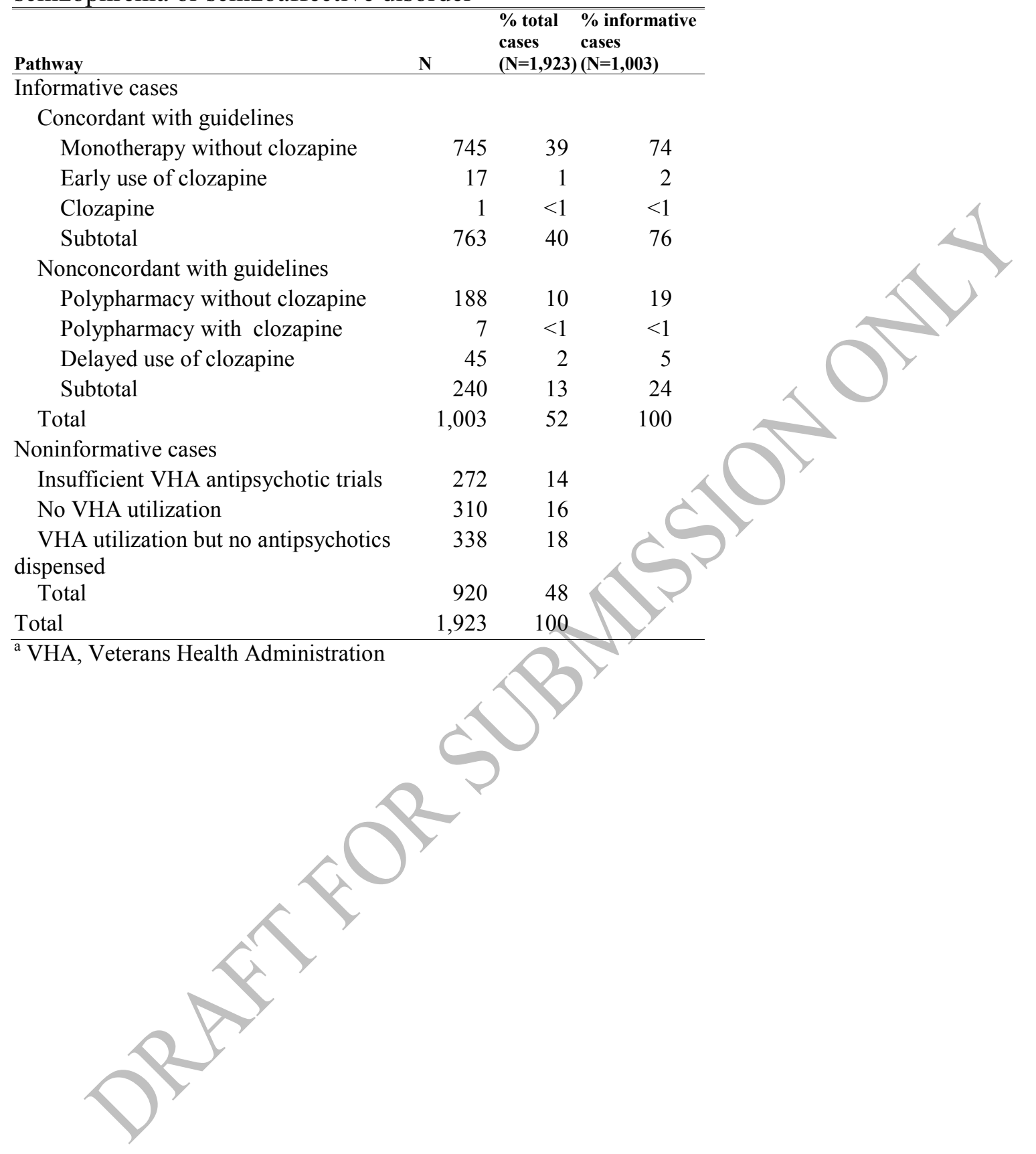


Table 2 Demographic and clinical characteristics of VHA patients with schizophrenia or schizoaffective disorder ${ }^{\mathrm{a}}$

\begin{tabular}{|c|c|c|c|c|c|c|}
\hline \multirow[b]{2}{*}{ Characteristic } & \multicolumn{2}{|c|}{$\begin{array}{l}\text { Total analytic sample } \\
(\mathrm{N}=933)\end{array}$} & \multicolumn{2}{|c|}{$\begin{array}{l}\text { Nonclozapine } \\
\text { monotherapy } \\
(\mathrm{N}=745) \\
\end{array}$} & \multicolumn{2}{|c|}{$\begin{array}{l}\text { Nonclozapine } \\
\text { polypharmacy } \\
(\mathrm{N}=188)\end{array}$} \\
\hline & $\mathbf{N}$ & $\%$ & $\mathbf{N}$ & $\%$ & $\mathbf{N}$ & $\%$ \\
\hline Caucasian & 258 & 54 & 193 & 52 & 65 & 65 \\
\hline Hispanic & 50 & 11 & 36 & 10 & 14 & 15 \\
\hline Male & 863 & 93 & 699 & 94 & 164 & 87 \\
\hline Not married & 417 & 45 & 332 & 45 & 85 & 46 \\
\hline \multicolumn{7}{|l|}{ Comorbidity $^{\mathrm{b}}$} \\
\hline Diabetes & 152 & 16 & 123 & 17 & 29 & 15 \\
\hline Hyperlipidemia & 156 & 17 & 115 & 15 & 41 & 22 \\
\hline
\end{tabular}

${ }^{\mathrm{a}}$ VHA, Veterans Health Administration. Because of missing data, the denominator for percentages is not the group maximum for all variables.

${ }^{b}$ Patients may appear in more than one category. 